\title{
Trade-offs in Miniature Quadrupole Designs
}

\author{
S. Boumsellek and R. J. Ferran \\ Ferran Scientific Inc., San Diego, California, USA
}

Pressing needs for miniature mass spectrometers became apparent during the last decade in process monitoring and control, space exploration, and environmental screening. Besides the small footprint, common requirements include low cost, low power consumption, field portability, reliability, autonomy, and ease-of-use. Design concepts and construction technologies of miniaturized quadrupole sensors were guided by cost reduction requirements without sacrifice of performance. The first miniature and complete quadrupole mass spectrometer system was introduced as the Micropole sensor. The concept featured a novel technique to assemble and operate multiple miniature quadrupoles in parallel. The short analyzer length offers a significant advantage by enabling direct mass filtering at pressures up in the $10^{-2}$ torr range. High voltages at higher frequencies $(10-20 \mathrm{MHz})$ are required for acceptable mass resolving powers. Additional trade-offs were uncovered in miniature sensors leading to designs optimized for each class of applications. Real time ray tracing of ions injected and filtered in the quadrupole field is used early in the design stage to predict the performance and reliability of the device. (J Am Soc Mass Spectrom 2001, 12, 633-640) (C) 2001 American Society for Mass Spectrometry

$\mathrm{M}$ ass spectrometers have evolved into standard tools capable of providing qualitative and quantitative analyses of the elemental, chemical, and isotopic composition of gases. Pressing needs for miniaturized versions in a wide range of applications became apparent early in the last decade. These applications cover many disciplines such as planetary exploration, residual gas analysis, and environmental monitoring. Besides the small size and low weight, common requirements include low cost, low power consumption, field portability, reliability, autonomy for long periods of time, and ease-of-use. The latter includes the ease of extracting the information, as non mass-spectroscopists are liable to use these tools.

In space applications, miniature rugged mass spectrometer sensors are designed into lightweight spacecraft for planetary exploration to perform chemical analysis of atmospheric composition or evolved gases from rock samples. When combined with a tool causing mechanical deformation, miniature mass spectrometers are able to sniff out traces of organics and/or precursors emitted from the surface of a solid sample. Immediate needs are for miniature sensors capable of rapidly detecting local leaks of hazardous gases such as hydrogen on the launch pad of the space shuttle system, and leaks of gases such as ammonia, hydrazine, oxygen, and nitrogen from the space station system during extra vehicular activity (EVA).

In semiconductor chip manufacturing, flat panel

Published online April 24, 2001

Address reprint requests to Dr. S. Boumsellek, Ferran Scientific Inc., 11558 Sorrento Valley Rd., San Diego, CA 92121. E-mail: Sparky@ferran.com display, and vacuum coating industries, small footprint, plug-and-play sensors are needed for easy upgrade and/or replacement in fabrication facilities. Such sensors are required to be an integral part for use as residual gas analyzers (RGAs) to qualify the process tool and as process gas analyzers (PGAs) to monitor thin film deposition processes. Networked together, these sensors are strategically mounted on cluster tools to monitor individual chambers and allow wafer-towafer fault detection schemes to be implemented to prevent scrap and enhance tool productivity.

In environmental applications, needs are for field deployable systems with lower vacuum requirement in numerous gas chromatography-mass spectrometry (GC-MS) applications. When combined with small vacuum pumps, battery operated instrumentation can be either hand carried, back packed, or carried by robots to monitor a variety of environmental conditions.

During the past decade, intense efforts were devoted to transforming mass spectrometers from bulky laboratory-type instruments to small, sensor-type devices. Among several types of mass spectrometers, linear quadrupoles were considered by many research groups as good candidates for dimensional down scaling because of their inherent simplicity and compactness. While different design concepts and construction technologies of miniaturized quadrupole sensors were proposed, they were all guided by cost reduction requirements without sacrifice of performance. In 1990, Ferran Scientific Inc. (San Diego, CA, USA) introduced to the scientific community the first miniature quadrupole (4 $\mathrm{cc}, 25 \mathrm{~g}$ ) based on a groundbreaking design called the 
Micropole sensor [1-3]. It consists of arrays of parallel quadrupoles assembled in a grid-like pattern using cylindrical rods $(0.5-1 \mathrm{~mm}$ in diameter, $2-20 \mathrm{~mm}$ long). A single ion source supplies all individual quadrupoles and filtered ions are collected on individual Faraday detectors all connected together. The rods are accurately aligned using photo-etched parts and secured using glass-to-metal sealing techniques in a high temperature oven. In addition, the glass structure includes electrical pins and serves as a vacuum seal for the sensor. Such a cost effective construction technique produces identical quadrupole arrays, and therefore interchangeable sensors, with regards to the driving RF circuitry. A larger device using a very similar design approach to that of the Micropole was later described by Orient et al. [4]. It consists of nine quadrupoles using rods $25 \mathrm{~mm}$ long and $2 \mathrm{~mm}$ in diameter. The rods are mounted on two machinable ceramic jigs, one at each end of the rods. Machined in each ceramic jig were 16 holes to align the rods, 9 additional metal plated holes for entry and exit of the ions, and four holes for mounting rods to sandwich the structure in place. Electrical contact was made at the back surface of the exit jig by spot welding strips to alternate rods. Holkeboer et al. [5] used a more conventional approach. Four hyperbolic rods, cut using electrical discharge machining (EDM) and bonded together with ceramic spacers, are assembled with an inscribed circle diameter of 660 $\mu \mathrm{m}$ making a mass filter $13 \mathrm{~mm}$ long. This assembly is attached to the ceramic base plate with a clamp. Leaf springs make electrical contact from the metal traces on the ceramic plate to the respective rods. Metallic traces on the ceramic plate connect the electrical feedthrough leads to the various sensor elements. Taylor et al. [6] developed a sensor based on a micro-electromechanical systems (MEMS) approach. The design features micromachined electrodes formed from metalized silica rods, mounted in pairs on two silicon substrates held apart by additional insulating cylindrical rods. The resulting rods are $500 \mu \mathrm{m}$ in diameter making mass filters 20-30 $\mathrm{mm}$ long. The sensor produced low-mass resolution over very limited mass ranges.

In addition to miniaturizing the quadrupole device, efforts were devoted to develop RF power supplies of comparable sizes. The short length of the mass filters of the different designs listed above requires generating high voltages at higher RF frequencies in order to provide suitable mass resolving powers. The closer proximity associated with the smaller dimensions of the poles of the miniature quadrupoles leads to increased capacitance. The increased frequency and capacitance poses challenges with regards to minimizing power losses when designing circuitry to fit in small, lightweight enclosures.

While miniature mass analyzers were recently reviewed by Badman and Cook [7], only linear quadrupole designs are discussed in the present paper. We highlight the benefits and challenges associated with the miniaturization of full size instruments from both the physical and the operational viewpoints. Performance trade-offs in miniature designs will be derived from the scaling laws. We present the performance characteristics obtained with different Micropole arrays. Finally, real time computer simulations performed during the design stage in an attempt to predict the performance and the reliability of the device are discussed.

\section{Benefits and Challenges of Miniaturization}

Quadrupoles are scanning devices and are more appropriately referred to as mass filters. Most conventional quadrupole designs feature four cylindrical rods (poles) assembled in a square fashion with an inscribed circle of radius $r_{0}$, which is a fraction (0.876) of the rod radius (r). This configuration is used to approximate the ideal hyperbolic fields with minimum fringing field distortions. The transmission of the quadrupole device is determined by the geometrical acceptance given by the sizes and elevation angles at the entrance and exit apertures of the filtering region. The mass filtering is accomplished by applying positive and negative combinations of dc and RF voltages on diametrically opposed rods. Scanning both dc and RF voltages while maintaining a constant ratio (or ac-to-dc ratio) of approximately 6 generates a mass spectrum. Conventional quadrupoles require a vacuum pressure below $5 \times 10^{-5}$ torr to operate in a linear mode. The upper pressure limit is given by the length $(\mathrm{L})$ of the rods.

The first trivial observation when scaling down the dimensions (by a factor $n$ ) of a charged particle device such as the quadrupole mass spectrometer is a smaller overall volume and surface area by $\mathrm{n}^{3}$ and $\mathrm{n}^{2}$, respectively. While the reduced volume adds to the overall robustness of the device, the reduced surface area of the sensor inlet leads to a total signal loss by a factor $\mathrm{n}^{2}$. The smaller overall surface area, however, reduces the memory effects and hence leads to faster response to gas concentration changes. Other benefits and challenges of miniaturization are derived from the dimensional down scaling applied to the equations, which govern the operation of a quadrupole device.

\section{Mass Resolution and Mass Range}

The two fundamental equations required for design purposes [8] are the mass resolution $\Delta \mathrm{M}(\mathrm{amu})$, defined here as the full width at half maximum (FWHM), of the mass peak and the mass range $\mathrm{M}_{\max }(\mathrm{amu})$ :

$$
\begin{aligned}
& \Delta \mathrm{M}=4 \times 10^{9} \mathrm{E}_{\mathrm{ion}} /\left(\mathrm{f}^{2} \mathrm{~L}^{2}\right) \\
& \mathrm{M}_{\max }=1.4 \times 10^{7} \mathrm{~V}_{\max } / \mathrm{f}^{2} \mathrm{r}_{0}^{2}
\end{aligned}
$$

where $V_{\max }$ is the maximum amplitude of the $R F$ voltage $(\mathrm{V})$ at frequency $\mathrm{f}(\mathrm{Hz})$, and $\mathrm{E}_{\text {ion }}$ is the ion 
extraction voltage $(\mathrm{V})$ from the ion source into the mass filter. $\mathrm{L}$ and $\mathrm{r}_{0}$ are in meters.

When scaling down the dimensions, maintaining the relative dimensional tolerances is crucial in quadrupole devices. Thus, to achieve a 0.5 AMU mass resolution over a 100 AMU mass range, a relative accuracy of about $0.2 \%$ is required $[4,8]$. An absolute accuracy of about $2 \mu \mathrm{m}$ is needed for ground stainless steel cylindrical rod $1 \mathrm{~mm}$ in diameter. Establishing a cost effective manufacturing technique could be challenging given that the precision required when assembling miniature parts could a priori be even more expensive compared to larger sensor components. Provided that the required dimensional tolerance and the alignment of miniature rods $(<10 \mathrm{~mm}$ long and $<1 \mathrm{~mm}$ in diameter) are met, filtering ions according to their mass-to-charge ratio $(\mathrm{m} / \mathrm{z})$ is accomplished by increasing the frequency of the RF fields (eq 1 and 2). Mass resolutions over mass ranges comparable to that of conventional size instruments are obtained by simply increasing the frequency by the same factor used for dimension down scaling (n). Thus, for a 10-mm-long filter, a mass resolution $\Delta \mathrm{M}=0.5 \mathrm{AMU}$ is expected when driving the filter at $\mathrm{f}=16 \mathrm{MHz}$ (eq 1). In order to cover a mass range from 0 to $100 \mathrm{AMU}$, for instance using 1-mm diameter rods, a power supply capable of delivering up to $1400 \mathrm{~V} p-p$ at $16 \mathrm{MHz}$ is needed. For a given mass resolution, using smaller diameter rods extends the mass range. Reducing the rod diameter by a factor of two extends the mass range by a factor of four at the same maximum RF voltage (eq 2). Generating such high voltages at such high frequencies in a small package could be a limiting factor as will be shown below. However, miniaturization offers a clear benefit vis-à-vis the likelihood of electrical discharges between adjacent rods. In fact, since mass filters operate well to the left of the minimum value ( 7 torr-mm for argon and tungsten) of the U-shaped Paschen voltage breakdown curve, decreasing the electrode gap increases the voltage required to cause a discharge.

\section{Upper Pressure Limit}

Due to the increased ion mean free path relative to the length of the mass filter of a miniature device, mass filtering at pressures much higher than the upper pressure limit of conventional instruments is enabled. The ions experience rather oscillatory trajectories inside the mass filter, meaning that the actual ion path is much longer than the length of the mass filter as it depends on the frequency. However, the upper pressure limit can be conservatively derived as being inversely proportional to the length of the quadrupole mass filter:

$$
\mathrm{P}_{\max } \alpha \mathrm{c} / \mathrm{L}
$$

For a 10-mm-long mass filter, the upper pressure limit $\mathrm{P}_{\max }$ is about $8 \mathrm{mtorr}$ (where $\mathrm{c}=8 \mathrm{mtorr}-\mathrm{cm}$ for argon for example). This characteristic is particularly advantageous in many applications as it eliminates the burden of differential pumping. Operating the quadrupole mass spectrometer directly at high pressures (mtorr range) extends the dynamic range of the device. Since minimum detectable pressures in $10^{-9}$ torr range are routinely obtained using simple Faraday-type detectors, sub-PPM sensitivity levels for trace gases are achievable. Furthermore, as a consequence of the high pressure operation where there is an overlap with other total pressure gauges, miniature quadrupole mass spectrometers are calibrated in the mtorr range using primary pressure transfer standards, such as capacitance diaphragm gauges, in order to provide absolute, partial, and total pressures. Exposing the hot filaments used for electron ionization to such high pressures could drastically reduce their lifetime depending on the temperature and the chemical nature of the ambient gases. Detecting corrosive gases, for instance, must be performed at lower pressures and hence over a reduced dynamic range, in order to obtain an acceptable filament lifetime. Less reactive sources of electrons such as cold cathode field emitter arrays were recently used as a source of electrons in a quadrupole mass spectrometer [9]. These devices are inherently small and showed promising results but their use is still limited to low pressures. Also, the choice of detectors becomes limited to Faraday cups at these higher pressures although a few vendors have recently introduced higher-pressure electron multipliers and micro-channel plates.

\section{Sensitivity}

The sensitivity or transmission of a quadrupole device is directly proportional to the geometrical acceptance (eq 4), which contains an area term (proportional to $\mathrm{r}_{0}^{2}$ ) and a solid angle term (proportional to $\mathrm{r}_{0}^{2} / \mathrm{L}^{2}$ according to Helmoltz-Lagrange law).

$$
\text { Acceptance } \alpha\left(\mathrm{r}_{0}^{2}\right)\left(\mathrm{r}_{0}^{2} / \mathrm{L}^{2}\right)
$$

The effect of scaling down the radius of the inscribed circle (as a consequence of scaling down the rod radius) and the length by the same factor $n$ cancels out in the solid angle term. However, the acceptance area term is reduced by $\mathrm{n}^{2}$, which leads to a signal loss by the same factor. The signal loss is recovered by assembling a two-dimensional ( $\mathrm{n}+1, \mathrm{n}+1)$ array of rods (of $\mathrm{r} / \mathrm{n}$ radius) making $\mathrm{n}^{2}$ (or a $\mathrm{n} \times \mathrm{n}$ array of) miniature quadrupoles [1-3]. The total acceptance area is now the sum of the individual acceptance areas (proportional to $\left(\mathrm{r}_{0}^{2} / \mathrm{n}^{2}\right)$ for each quadrupole. Quadrupole arrays were first suggested by Paul et al. [10] for better power efficiency, but the concept was used in the Micropole to recover the signal loss due to miniaturization without adding to the overall acceptance area (Figure 1). In addition, for a given acceptance area, the mass range 
can be increased by increasing the array size, which is accomplished by decreasing the rod diameter (eq 2).

\section{Power}

The power $\mathrm{P}$ required to generate high frequency RF voltages to drive quadrupole devices is estimated as the power drawn when transferring energy on and off a capacitor C of a RLC circuit:

$$
\mathrm{P}=C V_{\max }^{2} \mathrm{f} / \mathrm{Q}
$$

where $C$ is the capacitance between the rods of the quadrupole and $Q$ is the quality factor of the tuned circuit. In order to cover a mass range from 0 to 100 with 0.5 AMU resolution with a quadrupole mass filter using rods $1 \mathrm{~mm}$ in diameter and $10 \mathrm{~mm}$ long, voltages up to $1400 \mathrm{~V} p-p$ at $16 \mathrm{MHz}$ are needed. The power required to generate such voltages to drive capacitances of $40 \mathrm{pF}$ with $Q$ factor of 100 is about $13 \mathrm{~W}$. By assembling arrays of quadrupoles, a better power efficiency is accomplished since internal rods are common to multiple quadrupoles. The treatment below however, derives the power consumption as a function of the array size for a fixed total acceptance area (Figure 1).

\section{Conventional Quadrupole}

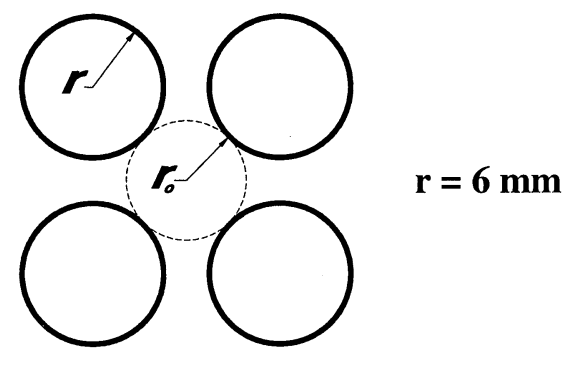

\section{Micropole Arrays}

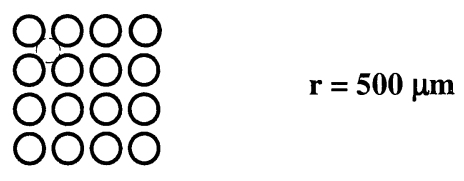

16 Poles, 9 Quadrupoles

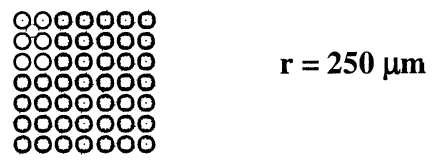

49 Poles, 36 Quadrupoles

Figure 1. Arrays of 1, 9, and 36 quadrupoles with a fixed total acceptance area.
In an array, the capacitances between individual rods are all in parallel, the total capacitance is the sum of all individual capacitances. Since the spacing between adjacent rods is proportional to the rod diameter, the individual capacitance is only proportional to the length of the rods. The total capacitance is thus proportional to the total number of rods $(n+1)^{2}$ and the rod length:

$$
\mathrm{C} \alpha(\mathrm{n}+1)^{2} \mathrm{~L}
$$

For a given acceptance area, $\mathrm{V}_{\max }$ is derived from eq 2 in the form:

$$
\mathrm{V}_{\text {max }} \alpha \mathrm{M}_{\text {max }} \mathrm{f}^{2} \mathrm{r}_{0}(\mathrm{n})^{2} \quad \text { where } \mathrm{r}_{0}(\mathrm{n})=\mathrm{r}_{0} / \mathrm{n} \quad \mathrm{n} \geq 1
$$

$r_{0}(n)$ is the radius of the inscribed circle, which depends on the down scaling factor $n . r_{0}(1)$ is the radius in the case of a single quadrupole; $(n+1)^{2}=4$ rods.

Hence the power is:

$$
\mathrm{P} \alpha\left((\mathrm{n}+1)^{2} \cdot \mathrm{L}\right) \mathrm{m}^{2} \cdot \mathrm{f}^{5} \cdot \mathrm{r}_{0}(\mathrm{n})^{4}
$$

For a fixed mass range, fixed frequency, and fixed length

$$
\mathrm{P} \alpha(\mathrm{n}+1)^{2} /(\mathrm{n})^{4}
$$

For large values of $n$, the power is inversely proportional to the quadrupole array size $\left(\mathrm{n}^{2}\right)$. When comparing a single quadrupole to a 16-rod array, the power saving $\left(\mathrm{P}_{16} / \mathrm{P}_{4}=4 / 81\right)$ is about $95 \%$. In the case of $16-$ and 49 -rod arrays, the power saving is $81 \%$.

\section{Micropole Arrays}

A full description of the Micropole device was given by Ferran and Boumsellek [3]. The system includes high frequency RF (7-20 MHz) power supplies, and a data acquisition system together with a computer interface to be connected to a PC through a serial port. A smaller package $(8 \mathrm{~cm} \times 8 \mathrm{~cm} \times 8 \mathrm{~cm})$, enclosing all electronic and power circuitry, was developed using switching power supplies, surface-mount components, and a self tuning RF power supply designed to minimize the power dissipation in the package (Figure 2).

The low cost manufacturing technique of the Micropole device was described by Ferran [2]. Briefly, metal rods and pins and glass structures are all mounted into a tool, which includes photo-etched plates to accurately align the rods and secure the pins into position. Since the rods are partially embedded in the glass, the total capacitance of built sensors is determined by both glass and air dielectric constants. Thus, the tooling allows building virtually identical mass filters making the sensors interchangeable vis-à-vis the driving RF circuitry. In addition, sensors with rods of different length and diameter are built with only minor tooling changes.

Tests of sensitivity, mass range, and resolution were 


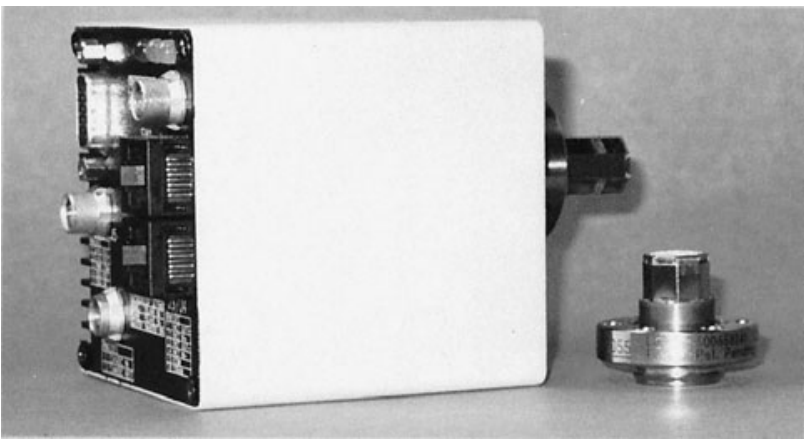

Figure 2. Complete system featuring sensor mounted on a mini Conflat $^{\mathrm{TM}}$ flange and electronics $(8 \mathrm{~cm} \times 8 \mathrm{~cm} \times 8 \mathrm{~cm})$.

obtained using Micropole arrays of different sizes (16 and 49 rods), rods of different lengths $(5-20 \mathrm{~mm})$ driven at different $\mathrm{RF}$ frequencies $(7-18 \mathrm{MHz})$ at pressures from $10^{-9}$ to $10^{-2}$ torr. Absolute intensities of the mass peaks were derived from calibrating the arrays (in the mtorr pressure range) against 1-torr capacitance diaphragm gauges. Typical mass spectra of inert gases obtained with arrays using mass filters $10 \mathrm{~mm}$ long are shown on Figure 3. Krypton isotopes $(m / z=78,80,82$, 83, 84, and 86) are measured with 3/4 AMU (FWHM) resolution at $14 \mathrm{MHz}$. Neon isotopes (displayed in a logarithmic scale) at $m / e=22,21$, and 20 in presence of residual moisture in the vacuum chamber are measured at $18 \mathrm{MHz}$. Three adjacent peaks are located at $m / e=16$, 17 , and 18 corresponding to $\mathrm{O}^{+}, \mathrm{HO}^{+}$, and $\mathrm{H}_{2} \mathrm{O}^{+}$ions respectively. All peaks are resolved with a width of 0.5 AMU (FWHM). The abundance sensitivity or mass rejection, for a $10-\mathrm{mm}$ mass filter driven at $18 \mathrm{MHz}$ is about 300 for adjacent masses. Thus, 10-ppm sensitivity levels to a trace gas are measured in the presence of a dominant gas separated by only two AMU units. If the dominant gas and the trace gas are separated by at least three AMU's, the abundance sensitivity is no longer the limiting factor but rather the absolute limit of detection (LOD) of the device.

The dynamic range and the linearity shown on Figure 4 are obtained using a 16-rod array of rods 15 $\mathrm{mm}$ long. The upper pressure limit is around $5 \times 10^{-3}$ torr while a LOD of $2 \times 10^{-9}$ torr is obtained with an electrometer dwell time of $25 \mathrm{msec}$ for the mass-tocharge of interest (argon). Operating the array at $5 \times$ $10^{-3}$ torr of a gas matrix, trace species in the sub-ppm level are measured. Lower amounts of trace species are measured using longer dwell times, and faster update times are obtained by only scanning the $\mathrm{m} / \mathrm{z}$ of interest. Finally, mass range capabilities of the miniature array were demonstrated using larger sample molecules such as n-docosane $(\mathrm{m} / \mathrm{e}=310 \mathrm{AMU})$ and 1-Docosanol $(\mathrm{m} / \mathrm{e}$ $=326 \mathrm{AMU})$ using 16- and 49-rod arrays [11, 12].

\section{Ray Tracing}

The performance of a quadrupole device such as the Micropole is determined by both geometrical (ion op-
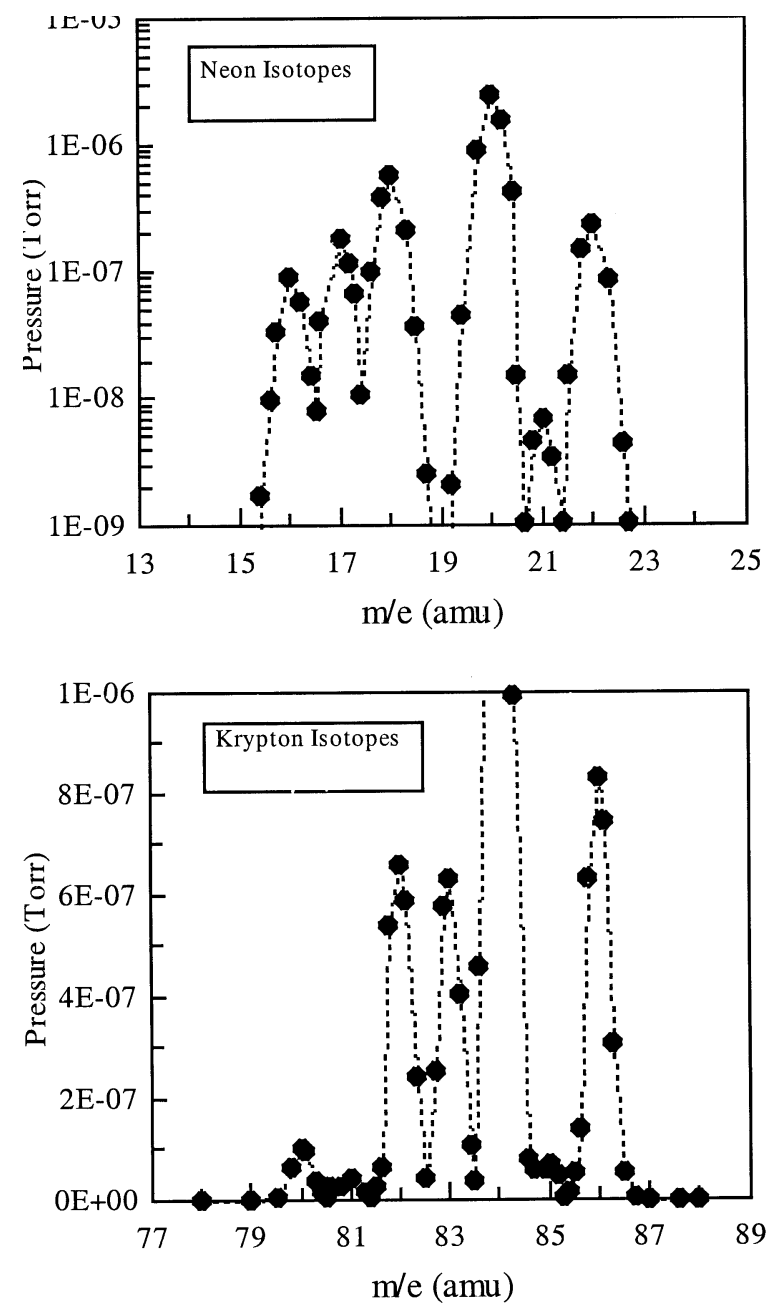

Figure 3. Neon and Krypton isotopes using arrays 10-mm long driven at $18 \mathrm{MHz}$ and $14 \mathrm{MHz}$ respectively.

tics, acceptance, and mechanical precision) and electrical parameters. The electrical or operating parameters consist of voltages applied to multiple electrodes to set the electron energy and emission, the ion energy, and the ac-to-dc ratio. For a given geometrical configuration, quadrupole mass spectrometers are able to electrically control performance characteristics such as mass range, mass resolution, sensitivity, and upper pressure limit. Since these characteristics are all interdependent, they are subject to trade-offs determined by the operating parameters. Any fluctuations of the electrical voltages associated with the operating parameters will affect the potential map seen by the ions and therefore will affect the stability of the device. To reduce the criticality of the fluctuations of the electrical voltages, the geometrical acceptance of the Micropole device must be optimized through computer simulations. Using the SIMION (Idaho National Engineering Laboratory, Idaho Falls, ID) code [13], real time ion trajectories in the mass filter were calculated for different ion injection conditions (axial offset, angular elevation, and injection velocity) at a given RF frequency. Figure 5 


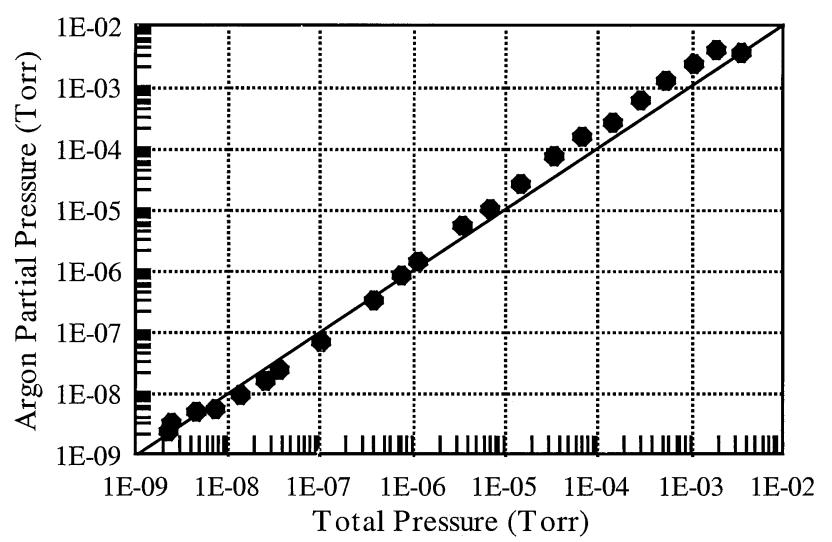

Figure 4. Dynamic range obtained using arrays $15 \mathrm{~mm}$ long and driven at $11 \mathrm{MHz}$.

shows a 2-D ( $\mathrm{x}, \mathrm{z}$ plane) cut of ion trajectories in a $(3 \times$ 3) array of quadrupoles. The simulated rods, $10 \mathrm{~mm}$ long and $1 \mathrm{~mm}$ in diameter are tuned for molecular nitrogen ions in an $18 \mathrm{MHz} \mathrm{RF}$ field. The ions are generated in the ion source, accelerated to a kinetic energy of $6 \mathrm{eV}$ with a $10 \%$ variation, and injected with axial velocities at random offset positions and elevation angles. All simulations were performed in cylindrical symmetry.

\section{Method}

By closing selected inlet apertures of a few quadrupoles and measuring the total output signal, experimental evidence showed no cross talk between the individual quadrupoles of the Micropole array. It was also dem- onstrated that the ion source is equally supplying individual mass filters with ions. Furthermore, ray tracing showed that ions outside the stability region of a quadrupole cannot enter an adjacent quadrupole and be confined within the stable region. Thus, a single quadrupole was sufficient to simulate the entire Micropole. The method used carefully analyzes the electrode configuration, through an iterative approach, to determine the ion initial conditions which lead to the smoothest dependence between mass resolution and sensitivity, and hence reduces the criticality of the influence of the electrical parameters. The actual relationship between resolution and sensitivity is complex as it depends on the current of the ion beam leaving the source, its divergence, and the defocusing action of the fringing fields between the ion source and the mass filter. In the following, ray tracing is used in an attempt to answer some design questions regarding the tradeoffs between sensitivity and resolution for different acceptances (geometrical considerations) and different ac-to-dc ratios (electrical considerations).

\section{Acceptance, Mass Resolution, and Sensitivity}

The number of ions extracted from the ionization region and injected into the mass filter depends on the geometrical acceptance: the area of the inlet aperture, and the solid angle determined by the entrance electrostatic lenses. For a given RF frequency, the amplitude of the ion oscillations in the filter depends upon the acceptance and the ac-to-dc ratio of the dc and RF voltages applied to the rods. The Micropole was tuned for $\mathrm{m} / \mathrm{e}=$ $28 \mathrm{AMU}$ and trajectories of hundreds of ions were

\section{Array of Mass Filters}

\section{Inlet Apertures}

Exit Apertures

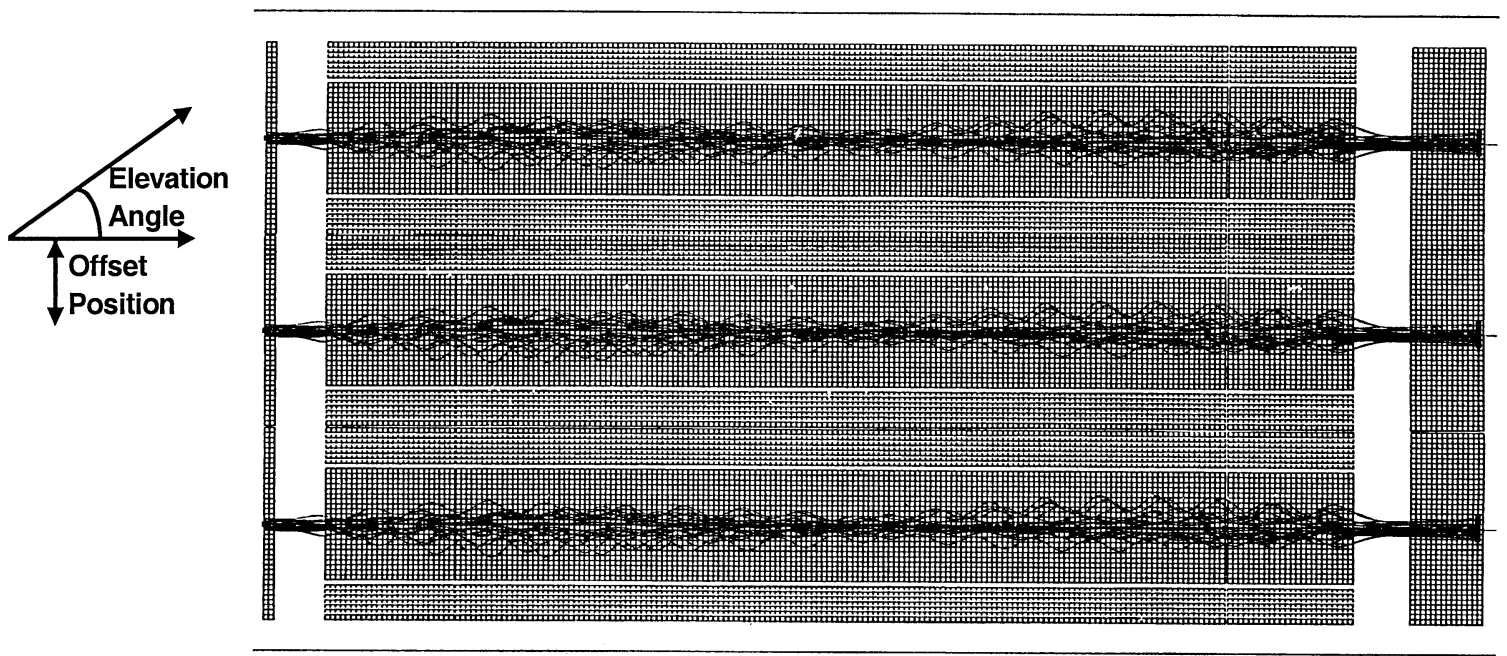

Figure 5. Two-dimensional cut ( $\mathrm{x}, \mathrm{z}$ plane) of stable ion trajectories in $18 \mathrm{MHz}$ RF fields in a $3 \times 3$ array of miniature quadrupoles (poles $10 \mathrm{~mm}$ long). 
calculated for different combinations of elevation angles and positions. Filtered ions are counted and their ratio to the total number of injected ions is called transmission or sensitivity. Geometrical acceptances for three different transmission ratios were computed and are shown on Figure 6. For a transmission greater than $95 \%$, the geometrical acceptance is an ellipse with a maximum angle of $5^{\circ}$ at the entrance aperture of the mass filter and an area with a radius $27 \%$ that of the inscribed circle. The transmission decreases rapidly to $50 \%$ outside the core ellipse when the elevation angle is increased by a few degrees. Ions with elevation angles of $10^{\circ}$ and higher are transmitted with less than $20 \%$ ratio (Figure 6).

The results above were obtained for the low-pressure end. As the pressure increases, non-linear effects inside the ion source, the mass filter, and at their interface lead to changes in the sensitivity of the device. In fact, due to the larger electron and ion densities in confined spaces, such as small diameter apertures, space charge effects alter the trajectories of charged particles. For a given diameter, the maximum ion density is determined by the onset of the ion repulsion due to space charge effects. Thus, pressure-dependent simulations were performed up to the mtorr pressure range.

For a given injection velocity, electron and ion charge densities, the ion optics (lenses and apertures at the inlet and exit of the mass filter) were optimized for maximum transmission and mass resolution with minimal ion losses due to space charge effects. While the size of the inlet aperture and the spacing of the entrance optics were specified according to the dimensions of the core ellipse obtained at the low pressure-end, the size of the exit aperture was determined when $5 \%$ of the injected ions are lost at the inlet aperture due to space charge effects. The calculated ion density corresponds to an ambient pressure around 2 mtorr [11]. At the space charge threshold, the diameter of the exit aperture was determined to be $82 \%$ of the diameter of the inscribed circle. Using this configuration, the mass resolution was determined to be 0.4 AMU (FWHM).

For the electrical configuration, the sensitivity versus the resolving power $\left(\mathrm{M}_{\max } / \Delta \mathrm{m}\right)$ was determined by varying the ac-to-dc ratio while maintaining a single ion-energy $(6 \mathrm{eV})$. Figure 7 shows a plot of the transmission between $15 \%$ and $100 \%$ versus the resolving power from 40 to 140 . The ac-to-dc ratio is increased from its optimum value (5.661) for maximum resolution to 5.958 for maximum transmission with a 0.02 step. The graph shows a relatively smooth transition between full transmission and maximum resolution. Experimental data obtained with sensors built according to the specifications determined by the simulations show a fairly good agreement (Figure 7).

\section{Conclusions}

Quadrupole mass analyzers are among a few charged particle devices that are suitable not only for miniatur-
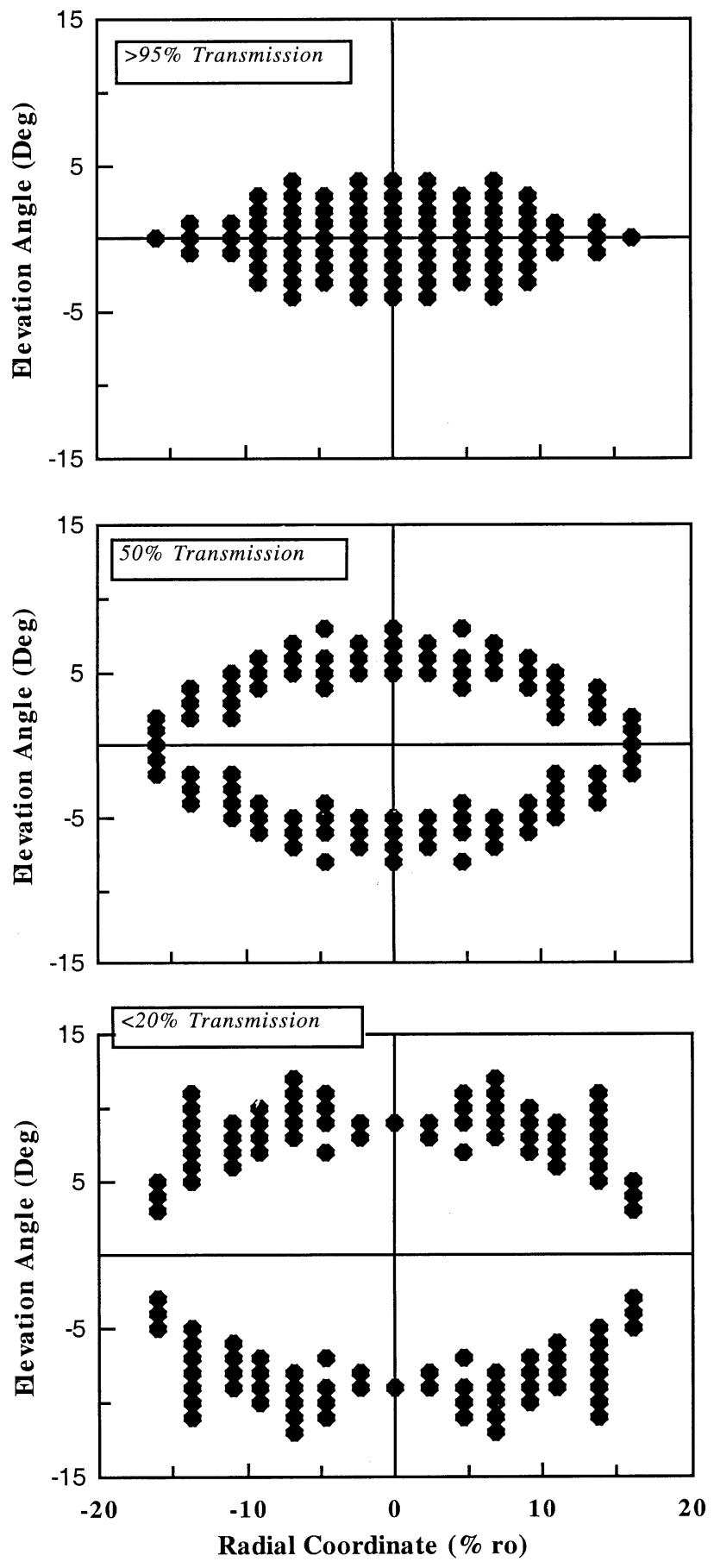

Figure 6. Geometrical acceptances for three different transmission ratios $(>95 \%, 50 \%,<20 \%)$.

ization but also for multiplexing or arraying. By providing a cost effective manufacturing technique capable of delivering the required accuracy and precision of handling miniature electrodes (poles, lenses, apertures etc...), performance comparable to that obtained with full size quadrupoles is achieved. The dimensional down scaling highlighted additional trade-offs engendered by the ability of miniature devices to operate at much higher pressures. The resulting extended dy- 


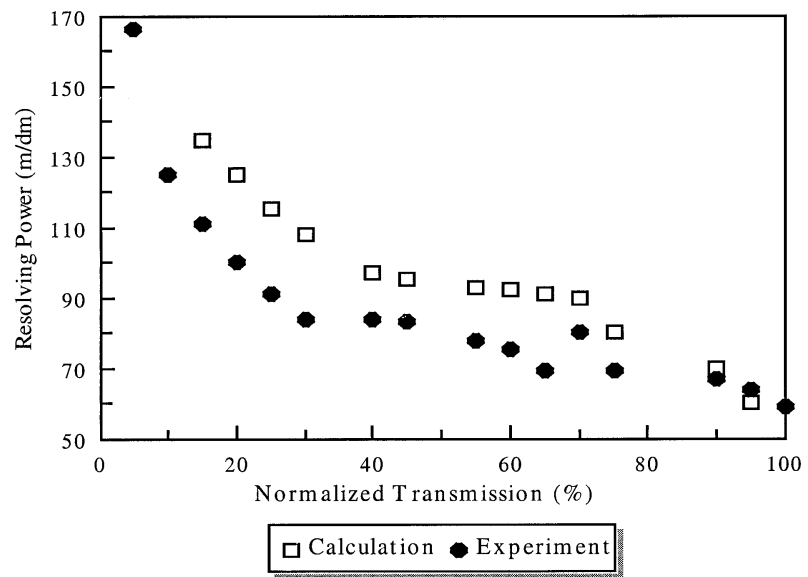

Figure 7. Resolving power $\left(\mathrm{M}_{\max } /(\Delta \mathrm{m})\right.$ versus normalized ion transmission obtained with ac-to-dc ratios ranging from 5.661 to 5.958 .

namic range leads to sub-ppm sensitivity levels using simple Faraday detectors. Performance characteristics such as mass resolution, mass range, upper pressure limit, and sensitivity are all interdependent through design parameters, e.g., rod diameter and length, frequency, and power requirements. Arrays of quadrupoles offer power saving and sensitivity advantages. When combined with a flexible construction technique accommodating sensors with different design parameters, the performance is tailored according to the specifications of the application. Predictions of the performance and reliability of the device was accomplished using real time computer simulations. By optimizing the interface between the static fields (at the inlet and exit of the mass filter) and the time-dependent quadrupole fields as a function of the ambient pressure, the criticality of the changes in the electrical parameters is reduced.

\section{Acknowledgments}

This work was partially funded, under contract (NAS10-98026), by the National Aeronautics and Space Administration. We would like to acknowledge Michael Oswald's (Design Engineer) contributions to the performance of the contract.

\section{References}

1. Ferran, R. J. U.S. Patent. 1995, 5,401,962.

2. Ferran, R. J. U.S. Patent. 1999, 5,857,890.

3. Ferran, R. J.; Boumsellek, S. J. Vac. Sci. Technol. A 1996, 14, 1258.

4. Orient, O. J.; Chutiian, A.; Garkanian, V. Rev. Sci. Instrum. 1997, 68, 1393.

5. Holkeboer, D. H.; Karandy, T. L.; Currier, F. C.; Frees, L. C.; Ellefson, R. E. J. Vac. Sci. Technol. A 1998, 16, 1157.

6. Taylor, S.; Tunstall, J. J.; Leck, J. H.; Tindall, R. F.; Jullien, J. P.; Batey, J.; Syms, R. R. A.; Tate, T.; Ahmad, M. M. Vacuum 1999, 53, 203.

7. Badman, E. R.; Cooks, R. G. J. Mass. Spectrom. 2000, 35, 659.

8. Dawson, P. H. Quadrupole Mass Spectrometry and its Applications; Elsevier: Amsterdam-Oxford-New York, 1976 p 125.

9. Felter, T.E. J. Vac. Sci. Technol. B 1999, 17, 1993.

10. Paul, W.; Reinhard, H. P.; Von Zahn, U. Z. Phys, 1958, 152, 143.

11. Boumsellek, S.; Ferran, R. J. J. IEST 1999, 42, 27.

12. Boumsellek, S.; Ferran, R. J. The 12th ASMS Sanibel Conference on Field Portable and Miniature Mass Spectrometry; Sanibel Island, FL, January, 2000; p 41.

13. Dahl, D. A. SIMION 3D Version 6.0. Report EGG-CS-7233, Idaho Nat. Eng. Lab., Idaho Falls, ID, 1995. 